\title{
Endoscopic agger nasi type Draf IIb treatment for frontal sinus lesions
}

\author{
LINGGAI SHI, JUN LIU, JIQING MA, FEI LIU and GUANGKE WANG \\ Department of Otolaryngology, Henan Provincial People's Hospital, Zhengzhou, Henan 450003, P.R. China
}

Received December 31, 2015; Accepted June 17, 2016

DOI: $10.3892 /$ etm.2016.3467

\begin{abstract}
Treatment of frontal sinus using surgery is complicated owing to the complex anatomical structure of the sinus region. The aim of the present study was to investigate the efficacy and safety of Draf IIb endoscopic frontal sinus surgery treatment for frontal sinus lesions using the agger nasi approach on 19 patients (28 left or and right nasal cavities). A $10-12 \mathrm{~mm}$ excision of the upper frontal maxilla was performed for endoscopic resection between the middle turbinate and lateral nasal wall. No serious complications in frontal sinus surgery treatment for the removal of the frontal sinus were observed. Patients were followed up after surgery for 6-36 months. Chronic sinusitis and nasal polyps were identified in 10 cases (19 left or and right nasal cavities; disease control, 15 left or and right nasal cavities; and disease partial control, 4 left or and right nasal cavities). Frontal sinus inverted papilloma was observed in 9 cases ( 9 left or and right nasal cavities). Frontal sinus inverted papilloma were successfully treated in 8 cases, and 1 case of recurrence was observed. In conclusion, the nasal endoscopic Draf IIb agger nasi approach is a minimally invasive treatment for frontal sinus lesions. This surgical procedure is safe and less complicated and may be applied in the clinic.
\end{abstract}

\section{Introduction}

Treatment of frontal sinus through surgery is complicated because of narrow frontal drainage channel and complex anatomical structure adjacent to the anterior skull base and screen of the artery at orbit board. Furthermore, the postoperative ostium of the frontal sinus leads to stricture or atresia and results in unsuccesful surgery (1). Therefore, the establishment of an optimal frontal sinus drainage channel is the purpose of frontal sinus endoscopic surgery.

In the present study, 19 cases underwent Draf IIb endoscopic frontal sinus surgery treatment for frontal sinus lesions using

Correspondence to: Dr Jun Liu, Department of Otolaryngology, Henan Provincial People's Hospital, 7 Weiwu Road, Zhengzhou, Henan 450003, P.R. China

E-mail: qz554m@163.com

Key words: agger nasi, Draf IIb, frontal sinus the agger nasi approach during the period November 2011 to June 2014. The results showed that Draf IIb minimal invasive treatment for frontal sinus treatment using the agger nasi approach provides a better result and safe surgical removal of frontal sinus lesions.

\section{Materials and methods}

General information. A total of 19 cases (28 left or and right nasal cavities) of frontal sinus lesion were diagnosed via endoscopic sinus surgery at the Henan Provincial People's Hospital during the period November 2011 to June 2014. Of the 19 cases, 13 male and 6 female cases of frontal sinus lesions were identified. Patients were aged 20-64 years, with an average age of 42 years, and the period of frontal sinus lesion was 6 months to 11 years. According to the criteria of Haikou (2), there were varying degrees of other sinusitis or nasal polyps associated with chronic frontal sinusitis in 10 cases. Seven cases with 13 left or and right nasal cavities of type II phase 3 and 3 cases with 6 left or and right nasal cavities of type III were also identified. The visual analogue scale (VAS) score was measured between 7 and 10 points. According to the criteria of Krouse (3), frontal sinus inverted papilloma of stage III was identified in 9 cases with 9 left or and right nasal cavities. The main clinical manifestations were a frontal headache, stuffy nose, purulent nasal discharge and pus. A preoperative horizontal position of computed tomography (CT) scan of nasal sinuses is shown in Fig. 1. The coronal and sagittal sinus were reconstructed. Antibiotics, and topical nasal spray hormone with oral steroids were administered to the patients, 7-10 days prior to surgery of chronic sinusitis. Oral mucus promoting discharge agent was administered and the nasal cavity was rinsed with physiological saline prior to surgery.

Surgery. The operation was performed under general anesthesia. A $0^{\circ}$ nasal endoscope (7230AA, Karl Storz, Tuttlingen, Germany) with conventional surgical resection and uncinate process between the middle turbinate and lateral nasal wall of the fornix was performed to remove the nasal dome surface mucosa. An incision of $\sim 10-12 \mathrm{~mm}$ height with $45^{\circ}$ of sphenoid sinus rongeur was made to completely remove the agger nasi posterior wall, a top wall and frontal surface. A resection of the frontal process of the upper maxilla to lamina papyracea plane was performed. To reach the anterior ethmoidal artery a $15-\mathrm{mm}$ incision was made from the front to the back up to the 


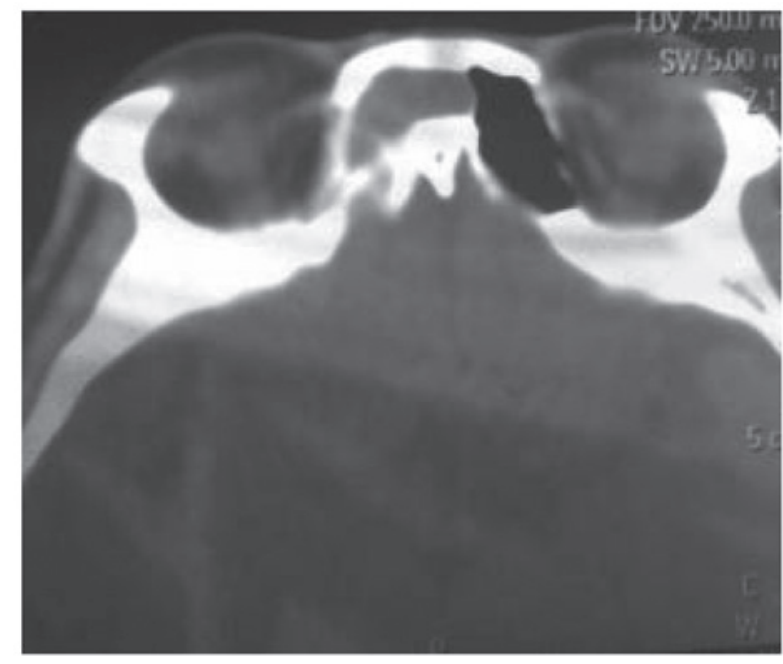

Figure 1. Preoperative CT scan of inverted papilloma of frontal sinus. CT, computed tomography.

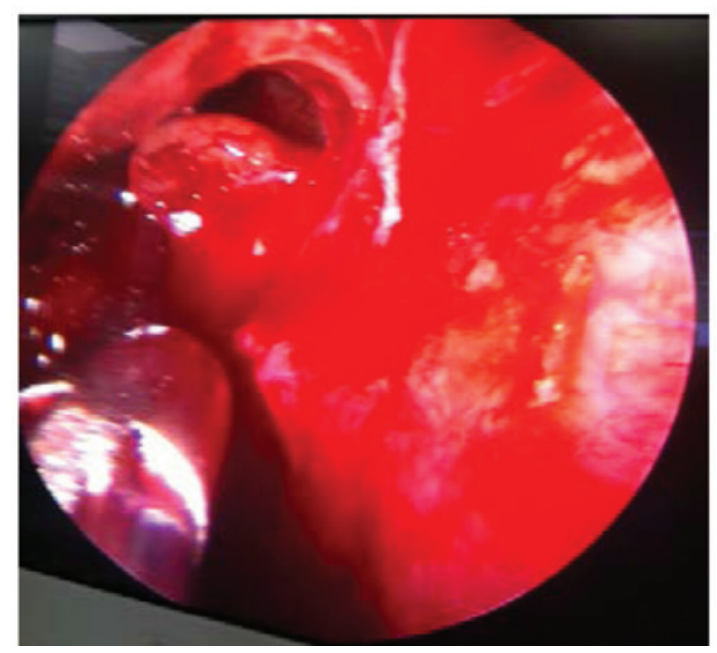

Figure 2. Intraoperative endoscopic view of inverted papilloma of frontal sinus.

middle turbinate before the attachment part. Subsequently, resection of the bottom wall of a frontal sinus of the middle turbinate and medial attachment up to the ostium of the frontal sinus medial to the nasal septum was performed. To expose the top and anterior walls of the frontal sinus, a section of the frontal ridges was ground using a frontal drill (1883672HS, Medtronic, Minneapolis, MN, USA). Frontal sinus lesions are shown clearly in Fig. 2. Fully open ostium of the frontal sinus is shown in Fig. 3. For the intraoperative treatment of frontal sinus inverted papilloma, drill grinding was employed with the exception of basal tumor bone and electric coagulation of the root. Hemostatic and anti-adhesive material were utilized as a rapid link.

Post-processing. After the chronic sinusitis operation, tropical nasal spray hormone and physiological saline were used to rinse the nasal cavity. Oral mucus promoting discharge agent, antibiotics, and oral steroid were also administered. First cleaning treatment was performed after 1-2 weeks of

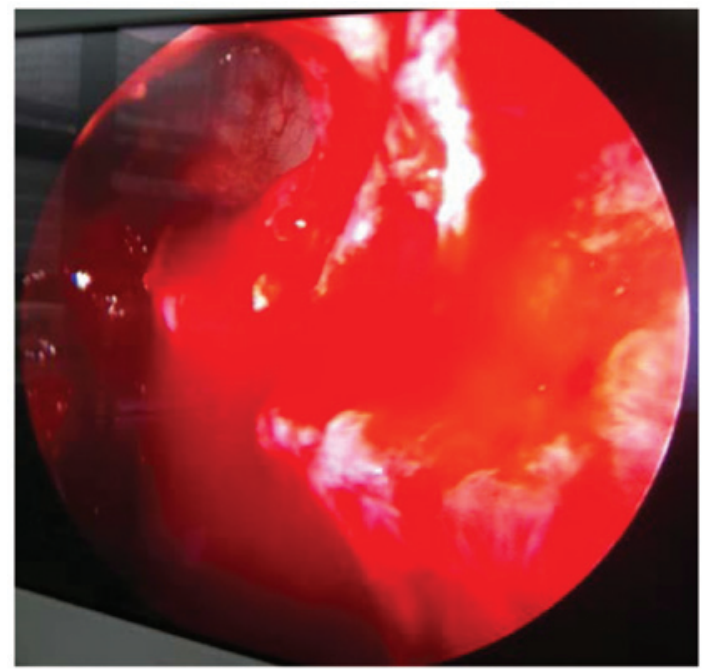

Figure 3. Postoperative endoscopic view of removed inverted papilloma for frontal sinus.

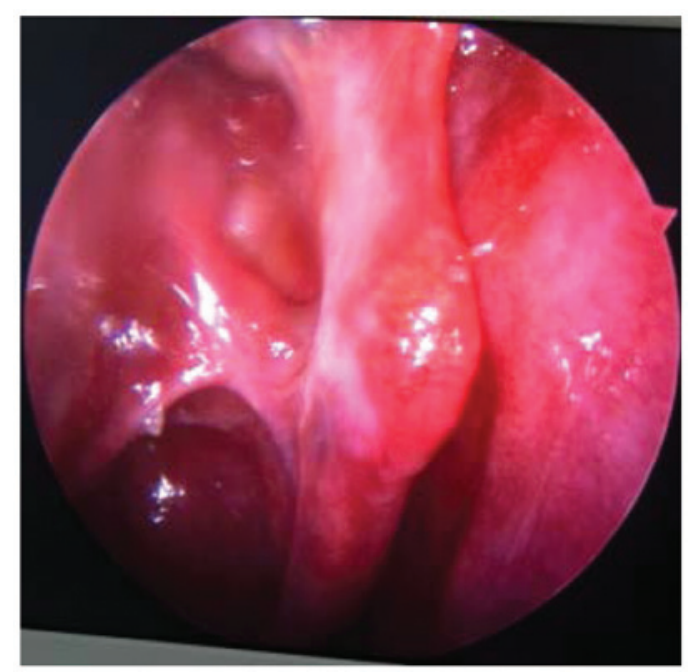

Figure 4. Seven-month postoperative nasal endoscopic view.

operating on the cavity. Suction was utilized to remove crusted debris and pseudomembrane. The subsequent operation cavity processing was conducted as per the recovery of operation cavity. Therefore, if an increased number of more vesicles, and granulation were identified the nasal cavity, review was conducted 2 weeks after the operation, whereas if the nasal cavity was relatively clean, a review was conducted once at 1 month or 2 months. The treatment interval was $<2$ weeks. At 2 weeks, if a bubble appeared it was removed by suction drainage. Any granulation was removed using cutting forceps (452831 or 452832, Karl Storz, Tuttlingen, Germany). Regular nasal endoscope operative cavity cleaning with mitomycin anti-adhesion was performed at the ostium of the frontal sinus and middle turbinate surface until the sinus and nasal cavity. Briefly, a cotton piece with $0.4 \mathrm{~g} / \mathrm{l} \mathrm{MMC}$ was placed for $5 \mathrm{~min}$, and then rinsed thoroughly with $200 \mathrm{ml}$ physiological saline. The operative cavity was reviewed once under nasal endoscopy (Karl Storz). A cotton piece was placed on the wound surface with $0.4 \mathrm{~g} / 1 \mathrm{MMC}$ for $5 \mathrm{~min}$, and rinsed thoroughly 
Table I. VAS and Lund-Kennedy scores with chronic sinusitis and nasal polyps.

\begin{tabular}{lccc}
\hline \multirow{2}{*}{$\begin{array}{l}\text { Side no. } \\
\text { (side) }\end{array}$} & Preoperative & After operation & $\begin{array}{c}\text { Lund-Kennedy } \\
\text { (points) }\end{array}$ \\
\cline { 2 - 3 } 15 & $9.05 \pm 0.705$ & 0 & 0 \\
4 & $9.22 \pm 0.667$ & $5.11 \pm 0.782$ & $2.33 \pm 0.500$ \\
\hline
\end{tabular}

VAS, visual analogue scale.

with $200 \mathrm{ml}$ physiological saline, after 1-2 months of operation. Inverted papilloma patients were followed up for the period of 6 months to 3 years. The operative cavity was reviewed once under nasal endoscopy, after 1-2 months of operation, and once each month in the first 3 months. Subsequent reviews occurred once every 6 months up to 3 years.

Efficacy evaluation. The postoperative efficacy of chronic sinusitis and nasal polyps was assessed according to the Chinese ENT and Head and Neck Surgery Journal Editorial Committee of the Sinusitis clinical diagnosis and treatment guidelines (CPOS2012) standard (4).

As a complete control of symptoms, the VAS total score was between 0 and 1 points according to Lund and Kennedy (5). After endoscopic surgery, the sinus opening was good, and the mucosal edema disappeared. No sticky purulent secretions were evident. The epithelium is shown in Fig. 4. According to the control, the symptoms improved significantly but did not completely subside. The VAS total score was $<3$ points or $>3$ points, and the Lund-Kennedy total score was $>1$ point.

Formation of the regional edema through endoscopic examination was observed. Additionally, hypertrophy or granulation tissue were evident in the region of the sinus mucosa, and there was a small amount of adhesive or purulent secretion. If there was no difference in the VAS score prior to and after the treatment, and a total score of Lund-Kennedy was not significantly decreased, patients were examined again. Through endoscopic examination, it was identified that there was a swelling of the mucus membrane of the sinus cavity and the formation of polyps or the proliferation of connective tissue was observed. Additionally, more extensive adhesion, stenosis or occlusion of the sinus orifice, and sticky purulent secretion were identified. The curative effect of frontal sinus inverted papilloma were observed via nasal endoscopic examination. The granulation tissue was taken for pathology examination. According to the results of the pathology examination, recurrence of the frontal sinus was determined.

\section{Results}

We successfully performed surgery of the frontal sinus. Unilateral periorbital edema for chronic sinusitis and nasal polyp type II phase 3 in 2 cases after the operation were observed. We performed nasal cavity cleaning and administered packing material and hormone. The results showed that the edema subsided in 3 days, and there was no visual acuity and diplopia. There was no cerebrospinal rhinorrhea, anterior ethmoidal artery bleeding, or other complications.

Nasal endoscopy was performed between 6 and 36 months after the surgery. MMC was used in the patients with nasal endoscopy after surgery, and the adhesion was not found at follow-up of 6-36 months. We observed chronic sinusitis and nasal polyps of type II phase 3 of 7 cases with 13 left or and right nasal cavities, disease control with 11 left or and right nasal cavities and diseased partial control with 2 left or and right nasal cavities. We observed type III for 6 left or and right nasal cavities in 3 cases, disease control for 4 left or and right nasal cavities and partial disease control for 2 left or and right nasal cavities. Chronic sinusitis and nasal polyps were identified in 10 cases with 19 left or and right nasal cavities, disease control with 15 left or and right nasal cavities and disease partial control with 4 left or and right nasal cavities. The results of the VAS and Lund-Kennedy scores obtained are shown in Table I. Frontal sinus inverted papilloma were observed in 9 cases with 9 left or and right nasal cavities. Six cases with frontal sinus inverted papilloma underwent further surgery owing to recurrence. The surgery was successful in 8 cases and recurrence was identified for 1 case. We also found a recurrence for pre-operation diagnosis of inverted papilloma and the postoperative pathological study of inverted papillary cancer.

\section{Discussion}

There are two basic ways of endoscopic frontal sinus operation for the frontal recess. The first is from front to back, and the second way is from bottom-up, respectively. The hook process and the nasal dome are important anatomic signs (6). The bottom-up method is mainly for the upper attachment of the uncinate process to sinus location. When the upper end of the uncinate process is attached to the skull base or middle turbinate, the mouth of the frontal sinus in the uncinate process of the outer side of the upper end is explored, whereas when the uncinate process of the upper attachment to the lamina papyracea occurs, the frontal sinus mouth in the inner side of the uncinate process is explored first (7). We observed severe mucosal edema and local formation of polyps in patients with a history of nasal surgery. In those patients, anatomical landmarks are difficult to identify. Furthermore, the operation is difficult because the angle lens device cannot reach the lesion site. In this situation, complete removal of the lesion is difficult, and this can easily damage the nasal cavity and sinus mucosa.

Agger nasi is the gas in the front room of frontal recess, at the top of the front of the ethmoid bone on the lateral wall of the nasal cavity, located in the front of the uncinate process, and upper section of the attachment middle turbinate and nasal lateral wall (8). At the top is the frontal recess and frontal sinus, the front section is the process of the maxilla, the rear is the frontal recess and ethmoidal infundibulum, which is in the uncinate process, and laterally is the nasal and lachrymal bone (8). Bolger et al observed that the occurrence rate of agger nasi was $98.5 \%$ (9). Thus, the agger nasi is the constant volume recess air real.

Schaefer and Close (10) first reported the agger nasi approach of frontal sinus operation and its theoretical basis 
is that the posterior wall of the nasal dome is the anterior wall of the frontal recess. On the bottom wall of the frontal sinus, the top posterior wall of a nasal dome is opened, which then opens the frontal recess. Wormald (11) improved this process further. According to Wormald's process, the basic characteristic is at $0^{\circ}$ endoscopy between the middle turbinate and lateral nasal wall which forms a cutaneous, mucosal flap whereby the pedicle is in the medial. This process facilitates removal of the agger nasi anterior, medial wall, posterior wall and the top wall of the frontal sinus openings. In addition, the amount of root of the middle turbinate mucosa flap repairs the anterior frontal recess of the exposed bone surface. The utility model has the advantages of $0^{\circ}$ endoscopic surgery. The operation is relatively convenient, and the operative field is also well exposed. Furthermore, anatomical structure recognition is relatively simple because of the frontal recess which is relatively narrow and a nasal process of frontal bone dysplasia (12). The ostium frontal sinus stenosis in most cases is due to postoperative mucus membrane edema or hyperplasia of frontal recess stenosis and adhesion. In the agger nasi diameter open frontal sinus (13), middle turbinate upper and frontal recess or agger nasi real gas adhesion was caused by frontal sinus ostium stenosis or atresia.

In the study of Wormald (14), 64 patients underwent surgery for frontal sinus and 6 patients had middle turbinate front and lateral nasal wall adhesion. In the present study, there was no operative cavity adhesion following surgery, and previous findings $(13,15)$ have identified that the difference is mainly because of the application of mitomycin $\mathrm{C}$ after the operation of endoscopic nasal surgery. Mitomycin $\mathrm{C}$ acts as an anti-drug metabolism that can cross-link with DNA double helix molecules and destroys the structure and function of DNA (16). This ultimately inhibits cell proliferation and DNA replication. This process prevents fibroblasts from producing collagen material and reduces scar formation and granulation tissue hyperplasia and prevents tissue adhesion. In the present study mitomycin cotton with $0.4 \mathrm{~g} / 1$ was placed in the middle turbinate upper and frontal recess or agger nasi to prevent postoperative cavity adhesion. The mitomycin treatment was useful in wound healing. The results showed that use of $0.4 \mathrm{~g} / \mathrm{l}$ mitomycin 4 min after endoscopic nasal surgery significantly reduced the incidence of postoperative adhesions. Patients were followed up for 4 months after surgery and no side effect was observed (17). In the present study, two left or and right nasal cavities orbital edema appeared in two days and then five days after surgery; however, the edema gradually disappeared. This observation may be associated with the orbital board minor injury. One case of recurrence was found to be stage III frontal sinus inverted papilloma. In the present study, inverted papillary adenocarcinoma was identified after the postoperative pathology study. Through endoscopic resection, a case of recurrence was identified three months after surgery and then comprehensive treatment was followed up. The present study employed the agger nasi approach Draf IIb frontal sinus surgery. The mucosal flap was not preserved and resection of the fornix position (height, 10-12 mm) and excision of the frontal process of the upper maxilla were performed to allow entrance to the path of a more widened frontal sinus. Resection of the upper end of the middle turbinate and medial frontal sinus wall easily exposed the medial frontal sinus wall.
Resection of part of the nasal process of the frontal bone allows the frontal sinus and frontal recess increased unobstructed drainage without the production of a mucosal flap. This process is time efficient with regard to surgery and reduces the bleeding. By contrast, the production of a mucosal flap in nasal endoscopy causes bleeding, which leads to blood pollution causing the operation field to become unclear. The operation time is also prolonged. This process also reduces postoperative adhesion and cause obstruction in dressing and inspection of the frontal sinus. To avoid intracranial and intraorbital complications, the anterior cranial base and the anterior artery should be cleared.

In conclusion, the agger nasi approach Draf IIb frontal sinus surgery for frontal sinus disease rarely uses the angled endoscope and equipment. The operation is convenient with clear operation visual field and less bleeding. Operation time is also short with fewer complications. This approach of surgery is worthy of clinical application.

\section{References}

1. Han JK, Ghanem T, Lee B and Gross CW: Various causes for frontal sinus obstruction. Am J Otolaryngol 30: 80-82, 2009.

2. China Medical Branch Department of Otolaryngology Otorhinolaryngology Medicine Editorial Board. Clinical classification of chronic sinusitis and nasal polyp stage and the evaluation standard endoscopic sinus surgery: Haikou. Chin J Otorhinolaryngol 33: 134, 1998.

3. Krouse JH: Development of a staging system for inverted papilloma. Laryngoscope 110: 965-968, 2000.

4. Chinese Otolaryngology Head and neck surgery Magazine Editorial Committee of Rhinology Group, Chinese medical Association of Otolaryngology - head and neck surgery, nasal science group, chronic nasal. Sinusitis diagnosis and treatment guidelines (as of 2012, Kunming). Chin Otorhinolaryngol Head Neck Surg 48: 92-94, 2013.

5. Lund VJ and Kennedy DW: Staging for rhinosinusitis. Otolaryngol Head Neck Surg 117: S35-S40, 1997.

6. Luo Zhang, Bing, Zhou Wen Tong Ge, et al: Endoscopic frontal sinus surgery through agger nasi cell approach. Chin Otolaryngol Head Neck Surg 40: 493-497, 2005 (In Chinese).

7. Friedman M, Bliznikas D, Vidyasagar R, et al: Frontal sinus surgery 2004: Update of clinical anatomy and surgical techniques. Op Tech Otolaryngol Head Neck Surg 15: 23-31, 2004.

8. Stammberger HR, Kennedy DW; Anatomic Terminology Group. Paranasal sinuses: anatomic terminology and nomenclature. Ann Otol Rhinol Laryngol Suppl 167: 7-16, 1995.

9. Bolger WE, Butzin CA and Parsons DS: Paranasal sinus bony anatomic variations and mucosal abnormalities: CT analysis for endoscopic sinus surgery. Laryngoscope 101: 56-64, 1991.

10. Schaefer SD and Close LG: Endoscopic management of frontal sinus disease. Laryngoscope 100: 155-160, 1990.

11. Wormald PJ: The agger nasi cell: the key to understanding the anatomy of the frontal recess. Otolaryngol Head Neck Surg 129: 497-507, 2003.

12. Shi J and Lai Y: Frontal sinus, the key stone in current endoscopic period, needs more focus surgery. Chin J Ophthalmol Otorhinolaryngol 12: 75-77, 2012.

13. Loury MC: Endoscopic frontal recess and frontal sinus ostium dissection. Laryngoscope 103: 455-458, 1993.

14. Wormald PJ. The axillary flap approach to the frontal recess. Laryngoscope 112: 494-499, 2002

15. Metson R: Endoscopic treatment of frontal sinusitis. Laryngoscope 102: 712-716, 1992.

16. Gutierrez T and Hopkins C: Safe application of mitomycin C within the nasal cavity. J Laryngol Otol 125: 309-310, 2011.

17. Chung JH, Cosenza MJ, Rahbar R and Metson RB: Mitomycin C for the prevention of adhesion formation after endoscopic sinus surgery: a randomized, controlled study. Otolaryngol Head Neck Surg 126: 468-474, 2002. 